\title{
Breves Considerações Sobre a Atenção à Pessoa com Transtorno do Espectro Autista na Rede Pública de Saúde ${ }^{1}$
}

\section{Brief Considerations Regarding the Attention to Individuals With Autism Spectrum Disorder in the Public Health System}

\section{Breves Consideraciones Sobre la Atención a la Persona con Trastorno del Espectro Autista en la Red Pública de Salud}

\author{
Jeane A. M. R. Araujo ${ }^{2}$ \\ André B. Veras \\ Universidade Católica Dom Bosco
}

André A. B. Varella

Universidade Católica Dom Bosco

Instituto Nacional de Ciência e Tecnologia Sobre Comportamento Cognição e Ensino (INCT-ECCE)

\begin{abstract}
Resumo
O Transtorno do Espectro Autista (TEA) é uma desordem do neurodesenvolvimento, caracterizada por déficits na comunicação social e presença de padrões de comportamento repetitivos. Como uma condição usualmente crônica, o TEA normalmente requer atenção de equipes interdisciplinares por afetar o desenvolvimento de forma global. Recentemente, o Ministério da Saúde publicou dois documentos que estabelecem a linha de cuidado e as diretrizes para sua reabilitação na rede pública de saúde brasileira. O presente artigo caracteriza e analisa a linha de cuidado proposta e as abordagens terapêuticas recomendadas. A análise permitiu verificar que os documentos reafirmam que pessoas com TEA são indivíduos com os mesmos direitos de pessoas com deficiência, seu cuidado deve ocorrer de maneira multidisciplinar pela Rede de Atenção Psicossocial, mas faltou clareza quanto aos critérios de escolha das abordagens terapêuticas e o local em que estas seriam oferecidas. Algumas implicações para o tratamento do TEA são discutidas.
\end{abstract}

Palavras-chave: saúde pública, Sistema Único de Saúde, reabilitação, Transtorno do Espectro Autista

\begin{abstract}
Autism Spectrum Disorder (TEA) is a neurodevelopmental disorder, characterized by deficits in social communication and by the presence of repetitive patterns of behavior. As a chronic condition, TEAD usually requires attention from interdisciplinary teams as it affects development in many areas. Recently, the Brazilian Ministry of Health (MS) published two documents establishing the guidelines for attention and rehabilitation of people with TEA in the national public health system. This article aims to characterize and analyze these guidelines and the recommended therapeutic approaches. The analysis showed the recognition of the TEA as a condition with the same rights as people with disabilities. The care approach is essentially multidisciplinary and supported by the Psychosocial Attention Network, but the criteria for recommending the therapeutic approaches and the place where therapies would be offered are not clear. Some implications for TEA treatment are discussed.

Keywords: public health, Unified Health System, rehabilitation, Autism Spectrum Disorder
\end{abstract}

\section{Resumen}

El Trastorno del Espectro Autista (TEA) es un desorden del neurodesarrollo, caracterizado por déficits en la comunicación social y presencia de patrones de comportamiento repetitivo. Como una condición usualmente crónica, el TEA normalmente requiere atención de equipos interdisciplinares pues afecta el desarrollo de manera global. Recientemente, el Ministerio de Salud publicó dos documentos que establecen la línea de cuidado y las directrices para su rehabilitación en la red pública de salud brasileña. El presente artículo caracteriza y analiza la línea de cuidado propuesta y las intervenciones terapéuticas

\footnotetext{
${ }^{1}$ Este manuscrito é produto da dissertação de mestrado da primeira autora, orientada pelo terceiro autor, e desenvolvida no Programa de Pós-Graduação em Psicologia da Universidade Católica Dom Bosco (UCDB). A preparação do manuscrito recebeu o apoio do INCT (financiado pelo CNPq \#465686/2014-1 e FAPESP \#2014/50909-8).

2 Endereço de contato: Rua Taiobá, 487, Cidade Jardim, CEP 79040-640, Campo Grande, MS. E-mail: jeanearaujo@terra.com.br
} 
recomendadas. El análisis permitió verificar que los documentos reafirman que personas con TEA son individuos con los mismos derechos que personas con discapacidad, y su cuidado debe realizarse de manera multidisciplinar por la Red de Atención Psicosocial, pero faltó aclarar en lo que se refiere a los criterios de elección de las intervenciones terapéuticas y el local donde dichos tratamientos serían ofrecidos. También se discuten algunas implicaciones para el tratamiento del TEA.

Palabras clave: salud pública, Sistema Único de Salud, rehabilitación, Trastorno del Espectro Autista

\section{Introdução}

O Transtorno do Espectro Autista (TEA) é um transtorno do neurodesenvolvimento, marcado por comprometimentos nas habilidades de comunicação social e pela presença de padrões de comportamentos estereotipados, repetitivos e restritos. O conjunto de déficits que uma pessoa com TEA apresenta varia em graus de intensidade (podendo ser leve, moderado ou severo) e também de indivíduo para indivíduo (déficits diferentes em pessoas diferentes). Isso implica uma caracterização do TEA como um continuum ou espectro com ampla variação na forma como se apresenta clinicamente (APA, 2014).

A heterogeneidade observada nos casos de TEA é uma das características do transtorno que torna complexo os processos de diagnóstico e tratamento. O diagnóstico de TEA pode ser dado tanto para aqueles casos de indivíduos que são oralizados e escolarizados, mas que apresentam dificuldades em compreender regras sociais, ironias e que se interessam demasiadamente por poucos tópicos específicos, quanto para aqueles indivíduos que não desenvolveram a linguagem, que evitam constantemente a presença de pessoas, que apresentem rituais e comportamentos estereotipados constantemente ao longo do dia, e que necessitam de auxílio para se alimentar, se vestir, se banhar, entre outras necessidades básicas. Independente do grau, o TEA requer tratamento por trazer importantes prejuízos para a vida da pessoa e por se constituir como condição crônica.

Tomados em conjunto, os prejuízos advindos do TEA são especialmente relevantes para o contexto da saúde pública, visto seu caráter crônico e a prevalência de TEA na população estimada entre 1\% a 2\% (Hahler \& Elsabbagh, 2015). Apesar da ausência de dados oficiais brasileiros, se considerados tais números, a estimativa é de que exista no Brasil pelo menos dois milhões de pessoas diagnosticadas com TEA. Os casos de TEA demandam minimamente por serviços de saúde para a redução dos seus déficits característicos e outros possíveis quadros que normalmente o acompanham. Por exemplo, de acordo com Lai, Lombardo e Baron-Cohen (2014), indivíduos com TEA comumente apresentam comorbidades, tais como um desenvolvimento motor e de linguagem atípicos, deficiência intelectual associada (aproximadamente, $45 \%$ dos casos), TDAH (28\%-44\%), epilepsia (8\%-30\%), problemas gastrointestinais (9\%-70\%), distúrbios do sono (50\%-80\%), ansiedade (42\%-56\%), comportamentos de autoagressão e heteroagressão ( $50 \%$ e 68\%, respectivamente), entre outros.

Em um estudo realizado na Grã-Bretanha, estimou-se que o custo do TEA, ao longo da vida, é de mais de 2,5 milhões de dólares, incluindo despesas familiares, medicamentos e educação (Peters-Scheffer, Didden, Korzilius, \& Matson, 2012). Indivíduos com TEA precisam de serviços que ofereçam desde o diagnóstico precoce, tratamentos que promovam a sua habilitação e inclusão social, atenção com relação às possíveis comorbidades, além de suporte para seus familiares. Portanto, o autismo deve ser tratado como uma questão de 
saúde pública e deve ser enfrentado como tal, visto seus impactos sobre a rede de saúde e a necessidade de investimentos para sua atenção.

No ano de 2014, o Ministério da Saúde (Brasil, 2014) publicou um documento que estabeleceu diretrizes mais específicas para atenção à reabilitação de pessoas com TEA. Nele, constam informações sobre sinais de problemas de desenvolvimento e comportamentais do TEA, alguns instrumentos para o rastreamento do autismo, avaliações e classificações diagnósticas, e orientações sobre planos terapêuticos. Em síntese, o Ministério da Saúde forneceu orientações sobre como a rede de serviços deveria acompanhar a pessoa com TEA, desde o momento em que o risco de autismo é identificado até o diagnóstico e intervenções para habilitação/reabilitação. Em 2015, o Ministério da Saúde publicou outro documento, intitulado "Linha de cuidado para atenção às pessoas com Transtorno do Espectro Autista e suas famílias na Rede de Atenção Psicossocial do Sistema Único de Saúde" (Brasil, 2015). Este segundo documento, direcionado a gestores e profissionais, trouxe informações específicas quanto à organização da rede de atenção e as abordagens terapêuticas para a habilitação/reabilitação de pessoas com TEA no Sistema Único de Saúde (SUS), de modo a orientar a implantação de serviços de atenção a pessoas com TEA.

O presente artigo teve por objetivo analisar e discutir alguns aspectos dessas diretrizes e da linha de cuidado proposta pelo Ministério da Saúde (Brasil, 2014; 2015). Algumas possíveis consequências, em médio e longo prazo, são levantadas e discutidas considerando-se o tratamento para habilitação/reabilitação do TEA. Pretendeu-se, ao final do estudo, oferecer um panorama sobre a questão do tratamento do TEA no contexto do SUS e alguns possíveis desafios.

\section{O Contexto Histórico: A Reforma Sanitária e a Estruturação de uma Rede de Atenção à Saúde da Pessoa com TEA}

O sistema público de cuidados foi instituído na Constituição Federal de 1988, depois de um longo processo de reforma, em que a saúde passou a ser considerada como um direito do cidadão. Entretanto, até então, a saúde pública refletia um período de crise de recursos e, em termos de atenção, não se comprometia em assistir a toda população, uma vez que a saúde não era um dever do Estado.

Na década de 1970, instaurou-se uma preocupação com a "expansão de um bem-estar social", em um período de aumento da incidência de doenças marcadas pela pobreza. Eram necessárias ações de enfrentamento coordenadas aos serviços de saúde com articulações nacionais e locais, além de capacitação profissional (Paiva \& Teixeira, 2013). No ano de 1975, foi criado o Sistema Nacional de Saúde, que fortalecia os campos da saúde pública e oferecia uma base legal para a gestão de um sistema. O Programa de Interiorização de Ações de Saúde e de Saneamento caracterizou a expansão da rede primária, que aproximou a política brasileira dos princípios da Organização Mundial de Saúde (OMS) e facilitou o avanço da reforma sanitária.

Paiva e Teixeira (2013) expressam que a reforma sanitária é conhecida como um período de redemocratização no Brasil e que, apesar das dificuldades, virou uma realidade. O movimento resultou na formulação de leis, ao longo dos anos, que gradualmente foram afirmando direitos importantes. A VIII Conferência Nacional de Saúde, realizada em 1986, foi um 
marco na saúde brasileira, por ter sido palco de importantes discussões que estabeleceram o que seria o SUS. A Lei Orgânica de Saúde n. 8.080/90 (Brasil, 1990) consolidou os princípios de equidade, integralidade e universalidade.

Em 2001, a Lei n. 10.216/2001 (Brasil, 2001) intitulada: "Lei da Reforma Psiquiátrica", veio assegurar um sistema organizado e planejado de abordagem longitudinal e interdisciplinar, para a reabilitação psicossocial de indivíduos com transtornos mentais na própria comunidade. No ano seguinte, em 2002, era estabelecida a Política Nacional de Saúde da Pessoa com Deficiência, através da Portaria n. 1.060, de 5 de junho de 2002 (Brasil, 2002), que garante a essas pessoas o direito à saúde e à assistência integral e reabilitação, oportunizando maiores possibilidades de inclusão (Vargas, Dutra, Barcelos, Possuelo, \& Krug, 2016).

Em 2011, o Decreto n. 7.612, de 17 de novembro, instituiu o Plano Nacional dos Direitos da Pessoa com Deficiência - Plano Viver sem Limite (Brasil, 2013). Esse plano propõe a integração de diversas políticas e programas voltados às pessoas com deficiência. Nele, diretrizes importantes garantem ações inclusivas: a acessibilidade; o acesso à educação; à qualificação da pessoa com deficiência para o mercado de trabalho; a ampliação e acesso às políticas de assistência social; a melhoria do acesso a serviços de intervenção e reabilitação; garantias à habitação adaptável; entre outras. Entretanto, não existia um consenso se o autismo era ou não um tipo de deficiência (visto que muitos indivíduos com TEA não apresentam deficiência intelectual e/ou física como comorbidades), o que representava uma barreira importante para muitas pessoas com TEA em termos de acesso a serviços.

O entendimento legal de que o TEA era um tipo de deficiência ocorreu em 2012, com a Política Nacional dos Direitos da Pessoa com Transtorno do Espectro do Autismo (Brasil, 2012). As diretrizes ao atendimento das pessoas com TEA se pautam na intersetorialidade das políticas e ações de atendimento, na consulta da comunidade e na atenção integral. A atenção engloba ações que vão desde o diagnóstico até as formas de intervenção (habilitação/reabilitação), passando pela inserção no mercado de trabalho, orientação aos pais e responsáveis, e até incentivo na formação e capacitação de profissionais para o atendimento ao TEA. As políticas públicas definiam que a atenção ao TEA se daria dentro da Rede de Atenção Psicossocial (RAPS).

A RAPS oferece às pessoas com sofrimento mental e seus familiares diversas oportunidades de acesso a serviços de atenção, em seus contextos de desenvolvimento (Brasil, 2015). A RAPS é formada por componentes que disponibilizam serviços para atender às demandas específicas e demandas gerais. A RAPS é composta da Atenção Básica em Saúde, Atenção Psicossocial e Estratégica, Atenção de Urgência e Emergência, Atenção Residencial de Caráter Transitório, Atenção Hospitalar, Estratégias de Desinstitucionalização e Estratégias de Reabilitação Psicossocial.

Os serviços de Atenção Psicossocial e Estratégia contam com os Centro de Atenção Psicossocial (CAPS), que são centros de referência ao cuidado à saúde mental. Os CAPS operam de portas abertas ao acolhimento e oportunizam espaços de convívio na comunidade, distribuídos em diversos territórios. Os CAPS têm o importante papel de fazer a articulação dos serviços de saúde e da rede intersetorial. Os programas disponibilizados pelos CAPS podem oferecer ações voltadas à construção de autonomia e inserção social (Brasil, 2015) para quem assim o necessitar, incluindo indivíduos diagnosticados com TEA. 
De um modo geral, as políticas de saúde apresentaram avanços importantes ao longo dos anos no sentido de garantir o acesso à saúde às pessoas com todos os tipos de deficiências e transtornos mentais, estruturando uma rede de atenção que atenda suas diversas demandas, respeitando sua singularidade. As políticas públicas são pautadas na lógica de trazer esses indivíduos para o convívio social, com tratamentos integrados à comunidade, organizados em rede e de forma intersetorial, garantindo direitos de acesso ao mercado de trabalho e a tecnologias de reabilitação.

\section{A Atenção à Pessoa com TEA na Rede Pública de Saúde Brasileira}

A Rede de Cuidados à Saúde da Pessoa com Deficiência foi instituída pelo Ministério da Saúde (Brasil, 2012). O programa: "Viver sem Limite: Plano Nacional da Pessoa com Deficiência" (Brasil, 2013) reafirmou os princípios do SUS na atenção de pessoas com deficiência e TEA, favorecendo serviços de intervenções sobre as alterações cognitivas, sociais, de linguagem, de cuidados específicos e singulares de habilitação e reabilitação (Brasil, 2014; 2015). Assim, no âmbito do SUS, considerando especialmente o princípio da integralidade (Paim \& Silva, 2010), foi assegurado ao indivíduo diagnosticado com TEA o atendimento às suas demandas em sua totalidade, tanto nos aspectos biológicos quanto psicológicos e socioculturais. A pessoa com TEA, portanto, deve ser assistida no que tange aos comprometimentos diretamente relacionados aos sinais de autismo (e.g., dificuldades de comunicação e socialização, presença de comportamentos ritualísticos etc.), às eventuais comorbidades (e.g., dificuldades no desenvolvimento de habilidades de autonomia, distúrbios do sono, distúrbios alimentares, Lai et al., 2014) e também, sempre que necessário, por questões de saúde não relacionadas ao TEA especificamente (tais como tratamento de infecções, problemas respiratórios etc.).

$A$ atenção à pessoa com TEA, assim como para todos os outros usuários do SUS, tem na Atenção Básica $(A B)$ a sua porta principal de entrada. Na AB ocorrem as ações de acolhimento e de acompanhamento, do pré-natal ao desenvolvimento infantil. Esse ponto da RAPS é importante na identificação precoce dos sinais do TEA e no encaminhamento aos serviços especializados, como o diagnóstico, prevenção de agravos e serviços de reabilitação, através dos Núcleos de Apoio à Saúde da Família (NASF), que são componentes da AB. Um importante avanço nessa direção foi a Lei n. 13.438, de 26 de abril de 2017, que tornou obrigatório o uso de protocolos para a avaliação de riscos para o desenvolvimento nos primeiros dezoito meses de vida (Brasil, 2017). Medidas dessa natureza tendem a favorecer a identificação de crianças em risco de autismo, o diagnóstico e início precoce das intervenções.

De acordo com o Ministério da Saúde (Brasil, 2014), a atenção especializada deve ocorrer nos Centros Especializados em Reabilitação (CER), Serviços de Reabilitação Intelectual e Autismo, Centro de Atenção Psicossocial (CAPS) e outras instituições especializadas. Um dos principais desafios da atenção especializada é o de prover assistência para habilitação/reabilitação da pessoa com TEA em razão dos comprometimentos no desenvolvimento impostos pelo transtorno. Habilitar consiste em "tornar hábil", do ponto de vista da destreza/inteligência ou autorização legal (Brasil, 2010), enquanto o termo "reabilitar" se refere ao retorno a uma condição de habilitação pré-existente. Estratégias de reabilitação compreendem práticas que permitem conceituar processos e organizar o pensar sobre a forma de desenvolver novas habilidades ou habilidades perdidas. Assim, elas devem ser aplicadas nas dificuldades 
a serem tratadas, de acordo com a necessidade e compreensão da complexidade e da natureza do déficit (Wilson, 2002).

Com relação às terapias especializadas, o Ministério da Saúde (Brasil, 2015) caracteriza as seguintes linhas terapêuticas para o atendimento em reabilitação ao TEA: (1) tratamento clínico de base psicanalítica; (2) Análise do Comportamento Aplicada (ABA); (3) Comunicação Suplementar e Alternativa; (4) Integração Sensorial; e (5) Tratamento e Educação para Crianças com Transtornos do Espectro do Autismo (TEACCH). O documento ainda menciona outros três tipos de "tecnologias de cuidado": "acompanhamento terapêutico" e "apareIhos de alta tecnologia" (apesar de não se caracterizarem exatamente como abordagens terapêuticas, mas sim como recursos terapêuticos), além de "tratamento medicamentoso". No caso deste último, o documento não faz menção a nenhum medicamento ou classe de medicamentos específica.

As terapias especializadas para a habilitação/reabilitação da pessoa com TEA ocupariam um papel de destaque na redução dos sinais do TEA. Considerando a heterogeneidade do TEA, em termos de sinais e de possíveis comorbidades (Lai et al., 2014), os serviços de habilitação/reabilitação devem ser interdisciplinares, com o objetivo de reduzir os sinais de TEA e favorecer uma vida social autônoma do indivíduo, especialmente no que se refere aos aspectos da linguagem, socialização e atividades de vida prática (Brasil, 2014). Considerando o disposto pelo Ministério da Saúde, os CER, Serviços de Reabilitação Intelectual e Autismo, juntamente com os CAPS e outros institutos especializados, teriam a importante função de oferecer as terapias especializadas, se constituindo enquanto um espaço de atendimento contínuo, integral e articulado com a atenção básica (Brasil, 2015).

\section{Considerações sobre as Diretrizes e a Linha de Cuidado para a Atenção ao TEA na Rede Pública de Saúde}

Uma análise dos documentos publicados pelo Ministério da Saúde revela uma preocupação legítima em oferecer diversos tipos de tratamento para o TEA na rede de atenção. Esse aspecto da rede de cuidado é fundamental, visto que indivíduos com TEA apresentam demandas variadas que exigem atenção de diversos profissionais, mesmo que tais demandas não se apresentem simultaneamente em um mesmo período da vida do indivíduo. Todavia, no que tange às abordagens terapêuticas apresentadas (Brasil, 2015), não há clareza acerca dos critérios adotados para se recomendar certas abordagens em detrimento de outras. Por ser uma condição grave, crônica e que acomete diversas áreas do desenvolvimento do indivíduo (Volkmar \& McPartland, 2014), um importante ponto a ser considerado para o processo de habilitação/reabilitação é a eficácia de tais abordagens.

O próprio Ministério da Saúde (Brasil, 2014, p. 80) observa que "a escolha entre as diversas abordagens existentes considere sua efetividade e segurança e seja tomada de acordo com a singularidade de cada caso". Terapias que não resultem na redução dos sinais de TEA e em melhorias significativas na qualidade de vida do indivíduo podem trazer prejuízos importantes, como o desperdício de recursos financeiros e humanos. Os tratamentos pouco eficazes ou sem respaldo científico (com eficácia desconhecida) podem resultar em uma iminente sobrecarga dos serviços públicos de saúde mental, visto que a expectativa é que novos casos de autismo sejam identificados ao longo dos próximos anos, aumentando ain- 
da mais a demanda por atendimentos interdisciplinares. Entretanto, não são discutidos ou apresentados no documento estudos sobre a eficácia das abordagens elencadas.

De acordo com o documento publicado em 2014, o processo de reabilitação deve privilegiar o desenvolvimento da linguagem, socialização e habilidades de autonomia diária. Porém, os documentos publicados pelo Ministério da Saúde não se posicionam claramente a respeito dos locais em que seriam oferecidas as terapias especializadas nas abordagens terapêuticas mencionadas. A atenção especializada, que concentra diversas ações, acontece nos CAPS, CER, Serviços de Reabilitação Intelectual e outros institutos, ambulatórios e especialidades. Mas estariam todos esses dispositivos aptos a oferecer os atendimentos de reabilitação recomendados pelo Ministério da Saúde (Brasil, 2015)? Por exemplo, seria o CAPS o local adequado para oferta de intervenções com alto grau de especialização (tais como ABA, Integração Sensorial e TEACCH)? Ou algumas dessas terapias seriam oferecidas apenas no CER e Serviços de Reabilitação Intelectual, enquanto atendimentos clínicos, medicamentosos e atenção psicossocial em geral aconteceriam no CAPS e CAPS-i?

A escassez de informações sobre os papéis das instituições que compõem a rede na atenção ao TEA (em especial o CER e os Serviços de Reabilitação Intelectual e Autismo) pode dificultar o planejamento e a oferta de serviços de forma articulada, eficaz e organizada. Cabe destacar que o bom funcionamento da rede implica a oferta de serviços alinhados com as funções de cada instituição, considerando inclusive os recursos humanos e a infraestrutura disponíveis. Por exemplo, um profissional da terapia ocupacional qualificado poderia oferecer atendimentos especializados de Integração Sensorial em um CER; porém, em um CAPS, esse mesmo profissional atuaria de forma diferente (por ex., promovendo ações de reinserção social dos usuários), uma vez que o CAPS e o CER são dispositivos com funções diferentes na rede. No estudo de Lima, Couto, Delgado e Oliveira (2014), diversas preocupações sobre os atendimentos de pessoas com TEA em um CAPS-i foram reveladas, tanto por familiares de usuários quanto por profissionais. Os participantes expressaram dúvidas importantes sobre como seriam realizados os atendimentos, a frequência ideal, as linhas terapêuticas, a infraestrutura física adequada, entre outras. É exatamente nesse sentido que uma definição mais clara dos papéis de cada um desses dispositivos da rede e dos serviços por eles oferecidos poderia evitar confusões e favorecer uma melhor organização da rede por parte da gestão pública.

\section{Considerações Finais}

Apesar de posicionar-se claramente em favor de diferentes abordagens no cuidado da pessoa com TEA, fica aberta a questão sobre qual a eficácia das tecnologias de cuidado listadas pelo MS no que se refere ao processo de habilitação/reabilitação. A pluralidade de intervenções não necessariamente consiste em uma vantagem (Howard, Stanislaw, Green, Sparkman, \& Cohen, 2014; Dillenburger, 2014), especialmente quando não se tem informações (atestadas por estudos metodologicamente consistentes) sobre quais ganhos exatamente as abordagens terapêuticas podem oferecer aos usuários. Portanto, é fundamental analisar a literatura especializada sobre a eficácia das abordagens terapêuticas no processo de habilitação e reabilitação da pessoa com TEA, visto que a escolha por tratamentos eficazes pode diminuir a sobrecarga nos serviços de saúde. 
Wong et al. (2015) realizaram uma ampla revisão de literatura dos estudos que identificaram práticas baseadas em evidência no tratamento do TEA. Os resultados identificaram um total de 27 intervenções que apresentam eficácia no tratamento do TEA, de acordo com estudos que atenderam aos rigorosos critérios de qualidade. Das 27 intervenções baseadas em evidências, 16 delas são intervenções específicas de uma das abordagens recomendadas pelo Ministério da Saúde (ABA, Análise do Comportamento Aplicada). Outras sete intervenções são utilizadas pela ABA, TEACCH e Comunicação Suplementar e Alternativa. Os autores destacam que a ABA é a abordagem que apresenta maior suporte de estudos de alta qualidade, o que confirma resultados de outros estudos mais recentes (Eikeseth, Klintwall, Jahr, \& Karlsson, 2012; Howard et al., 2014; Roth, Gillis \& Reed, 2014). No entanto, também é necessário revisar de forma sistemática a literatura sobre a eficácia das outras abordagens terapêuticas, o que poderia ser realizado em estudos futuros.

O progresso da política de saúde brasileira é um fato concreto, que se consolida por medidas gradativamente implantadas para proporcionar um bem-estar e uma assistência integral e humanizada às pessoas com TEA. Esse fato torna-se mais importante quando se observa a preocupação por políticas que atendam às demandas das pessoas com TEA, de modo específico. Os esforços do Ministério da Saúde em organizar uma rede de atenção e oferecer serviços de reabilitação especializados são de grande relevância e se mostram coerentes com os princípios do SUS. Assim, é importante destacar e reconhecer esses avanços e esforços; mas também, reconhecer a necessidade de definir melhor os papéis das instituições envolvidas na atenção especializada e os serviços de reabilitação (baseados em evidências) que serão ofertados por profissionais qualificados para sua prática.

\section{Referências}

Associação de Psiquiatria Americana. (2014). Manual Diagnóstico e Estatístico de Transtornos Mentais (4a ed.). Porto Alegre: Artes Médicas.

Brasil. (1990). Lei n. 8.080, de 19 de setembro de 1990. Dispõe sobre as condições para a promoção, proteção e recuperação da saúde, a organização e o funcionamento dos serviços correspondentes e dá outras providências. Diário Oficial da União, Brasília, DF. Disponível em http://www.planalto.gov.br/ccivil_03/leis//8080.htm

Brasil. (2001). Lei n. 10.216, de 6 de abril de 2001. Dispõe sobre a proteção e os direitos das pessoas portadoras de transtornos mentais e redireciona o modelo assistencial em saúde mental. Diário Oficial da União, Brasília, DF. Disponível em http://www.planalto.gov.br/ ccivil_03/leis/leis_2001/l10216.htm

Brasil. (2002). Portaria n. 1.060 de 5 de junho de 2002. Diário Oficial da União, Brasília, DF. Disponível em http://bvsms.saude.gov.br/bvs/saudelegis/gm/2002/prt1060_05_06_ 2002.html

Brasil. Ministério da Saúde. (2010). Política Nacional de Humanização. Formação e intervenção. Brasília, DF. Disponível em http://bvsms.saude.gov.br/bvs/publicacoes/cadernos_humanizaSUS.pdf

Brasil. (2012). Lei n. 12.764, de 27 de dezembro de 2012. Institui a Política Nacional de Proteção dos Direitos da Pessoa com Transtorno do Espectro Autista e altera o § 3으 do art. 98 da Lei n. 8.112, de 11 de dezembro de 1990. Diário Oficial da União. Brasília, 
DF: Ministério da Saúde. Disponível em http://www.planalto.gov.br/ccivil_03/_ato20112014/2012/lei/l12764.htm

Brasil. Secretaria de Direitos Humanos. (2013). Viver sem limite. Plano Nacional dos Direitos das Pessoas com Deficiência. Brasília, DF. Disponível em http://www.pessoacomdeficiencia.gov.br/app/sites/default/files/arquivos/\%5Bfield_generico_imagens-filefield-description\%5D_0.pdf

Brasil. Ministério da Saúde. (2014). Diretrizes de Atenção a Reabilitação da Pessoa com Transtorno do Espectro do Autismo. Disponível em http://bvsms.saude.gov.br/bvs/publicacoes/diretrizes_atencao_reabilitacao_pessoa_autismo.pdf

Brasil. Ministério da Saúde. (2015). Linha de cuidado para a atenção às pessoas com transtornos do espectro do autismo e suas famílias na Rede de Atenção Psicossocial do Sistema Único de Saúde. Disponível em http://bvsms.saude.gov.br/bvs/publicacoes/linha_cuidado_atencao_pessoas_transtorno.pdf

Brasil. (2017). Lei Ordinária n. 13.438 de 26 de abril de 2017. Altera a Lei n. 8.069, de 13 de julho de 1990 (Estatuto da Criança e do Adolescente), para tornar obrigatória a adoção pelo Sistema Único de Saúde (SUS) de protocolo que estabeleça padrões para a avaliação de riscos para o desenvolvimento psíquico das crianças. Diário Oficial da União, Brasília, DF. Disponível em http://www2.camara.leg.br/legin/fed/lei/2017/lei-13438-26-abril-2017-784640-publicacaooriginal-152405-pl.html

Dillenburger, K. (2014). Multidisciplinary teamwork in autism: can one size fit all? The Australian Educational and Developmental Psychologist, 31, 97-112.

Eikeseth, S., Klintwall, L., Jahr, E., \& Karlsson, P. (2012). Outcome for children with autism receiving early and intensive behavioral intervention in mainstream preschool and kindergarten settings. Research in Autism Spectrum Disorders, 6, 829-835. doi: 10.1016/j. rasd.2011.09.002

Hahler, E., \& Elsabbagh, M. (2015). Autism: A global perspective. Current Developmental Disorders Reports, 2, 58-64.

Howard, J. S., Stanislaw, H., Green, G., Sparkman, C. R., \& Cohen, H. G. (2014). Comparison of behavior-analytic and eclectic early interventions for young children with autism after three years. Research in Developmental Disabilities, 35, 3326-3344. doi: 10.1016/j. ridd.2014.08.021

Lai, M. C., Lombardo, M. V., \& Baron-Cohen, S. (2014). Autism. Lancet., 383, 896-910.

Lima, R. C., Couto, M. C. V., Delgado, P. G. G, \& Oliveira, B. D. C. (2014). Indicadores sobre o cuidado a crianças e adolescentes com autismo na rede de CAPSi da região metropolitana do Rio de Janeiro. Physis Revista de Saúde Coletiva, 3(24), 715-739.

Paim, J. S., \& Silva, L. M. V. (2010). Universalidade, integralidade, equidade e SUS. Boletim do Instituto de Saúde, 12, 109-114.

Paiva, C. H. A., \& Teixeira, L. A. (2013). Reforma sanitária e a criação do Sistema Único de Saúde: notas sobre contextos e autores. História, Ciência e Saúde, 20, 15-35.

Peters-Scheffer, N., Didden, R., Korzilius, H., \& Matson, J. (2012). Cost comparison of earIy intensive behavioral intervention and treatment as usual for children with autism spectrum disorder in the Netherland. Research in Developmental Disabilities, 6(33), 1763-1772. 
Roth, M. E.; Gillis, J. M., \& Reed, F. D. D. (2014). A meta-analysis of behavioral interventions for adolescents and adults with autism spectrum disorders. Journal of Behavioral Education, 23, 258-286.

Vargas, S. C., Dutra, R. R., Barcelos, L. F. H., Possuelo, L. G., \& Krug, S. B. F. (2016). Assistência à saúde da pessoa com deficiência nos serviços públicos de saúde: um estudo bibliográfico. Revista de Epidemiologia e Controle de Infecção, 6, 224-234.

Volkmar, F. \& McPartland, J. C. (2014). From Kanner to DSM-5 - Autism as an evolving diagnostic concept. Annual Review of Clinical Psychology, 10, 193-212. doi: 10.1146/ annurev-clinpsy-032813-153710

Wilson, B. (2002). Towards a comprehensive model of cognitive rehabilitation. Neuropsychological rehabilitation, 2(12), 97-110.

Wong, C., Odom, S. L., Hume, K. A., Cox, A. W., Fettig, A., Kucharczyk, S., . . Schultz, T. R. (2015). Evidence-based practices for children, youth, and young adults with autism spectrum disorder: A comprehensive review. Journal of Autism and Developmental Disorders, 45, 1951-1966. doi: 10.1007/s10803-014-2351-z

Recebido: 04/08/2017

Última revisão: 20/11/2017

Aceite final: 14/02/2018

\section{Sobre os autores:}

Jeane A. M. R. Araujo: Mestre pelo Programa de Pós-Graduação em Psicologia da Universidade Católica Dom Bosco. E-mail: jeanearaujo@terra.com.br

André B. Veras: Docente do Programa de Pós-Graduação em Psicologia da Universidade Católica Dom Bosco. E-mail: barcielaveras@hotmail.com

André A. B. Varella: Docente do Programa de Pós-Graduação em Psicologia da Universidade Católica Dom Bosco. E-mail: andreabvarella@gmail.com 